\title{
Pautas de adquisición y uso del pronombre personal en lengua española. Un estudio longitudinal.
}

\author{
Grace Shum \\ Angeles Conde \\ Carmen Díaz \\ Universidad Complutense de Madrid

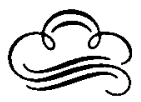 \\ Resumen
}

El estudio sobre los pronombres personales, constituye también una manera de investigar la adquisición del lenguaje y su desarrollo, dado que son categorías lingüísticas. El niño no adquiere el dominio de estas categorías tan pronto como lo bace con otros aspectos del lenguaje, aunque los usa desde muy temprana edad. De esta manera suponemos que al comienzo el niño utiliza las formas pronominales únicamente como dé́cticos. Esto es, sólo puede comprenderse el significado de estos términos en las expresiones infantiles con referencia al tiempo y al contexto comunicativo presente y concreto. Por esta razón autores como Clark, (1974, 1978), Chamey (1980), afirman que le pronombre personal, en su sentido lingüístico, no es adquirido por los niños basta los ocho o nueve años de edad.

En el presente estudio intentamos poner de manifiesto cómo adquieren los niños españoles estos términos y cómo evolucionan en su desarrollo. Para ello, se realizó un análisis de la producción verbal de varios niños, en sus aspectos: sintáctico, semántico y pragmático. Dicha producción se recogió a tra. vés de observación natural. Los sujetos de este estudio de medios ambientales diferentes.

Palabras clave: Pronombre, Deixis, Adquisición lingüística, Pragmática.

\section{Abstract}

The study about personal pronoun acquisition is not but just one of the maner to investigate the lenguage acquisition and development. It is thus because, as we are well known, that all the personal pronouns are linguistic categories. The child can not be able to get these categories acquisition as soon as be does with the other language categories, though be makes use them very early. However we may suppose that during the beginning, the child only makes use the personal pronoun on the deixis function. So be needs necesary to express it on the interpersonal communication (in the present time and throug the help of the communicative context). It is why that many investigators make sure (Clark, 1974, 1978; Chamey, 1980, etc.) that the personal pronoun, in a sense of linguitic function, cannot be acquired until eight or nine years of age.

The present study is about the Spanish language personal pronouns acquisition and development. We try to find out bow these categories are acquired by children. We observed: how they express these categories?, when the linguistic function of these pronouns are acquired?, bow is the process in chang. ing from deixis function to linguitic function?. It is a longitudinal and transversal study. We observed three children from two to three and balf years old.

Key words: Pronoum, Linguistic Acquisition, Pragmatic.

Dirección de la primera autora: Departamento de Psicología Evolutiva y de la Educación. Facultad de Psicología. Universidad Complutense de Madrid. Campus de Somosaguas. 28023 Madrid. 


\section{INTRODUCCION}

La adquisición del pronombre debe situarse en el ámbito del desarrollo del lenguaje y de la comunicación infantil. Su estudio entraña ciertas dificultades, debido a las características de esta categoría gramatical; comenzando por la propia definición del pronombre dentro de la lingüística. Existen divergencias entre los lingüistas y los semiólogos a la hora de señalar las características de estos términos, así como lo que debe o no debe ser incluido dentro de esta categoría. Jespersen (citado en Jasobson, 1975) afirma que los pronombres son comutadores (shifters), esto es, palabras que no pueden ser definidas sin hacer referencia o remitirse al mensaje, y cuya naturaleza semiótica las sitúa en un lugar intermedio entre símbolos e índices. Se trata de términos convencionalizados, que pueden ser definidos desde el ámbito de lo simbiótico, pero no pueden representar un objeto sin estar en relación existencial con él (Pierce, 1932). Para Lyons (1977), en cuanto deícticos, tienen un carácter primitivo, y aparecen antes que otras expresiones referenciales, y Humbold (citado en Jakobson, 1975) señala que son el estado más elemental y primitivo del lenguaje. Así pues, se pueden definir los pronombres, en un sentido general, como palabras gramaticales con una función nominal (Porto, 1986) y, en concreto, los pronombres más específicos o genuinos: los personajes - a los que hace referencia este estudio- se pue de decir que son términos cuya misión es señalar los protagonistas del coloquio o, en otras palabras, señalar las tres personas gramaticales. Así:

Primera. Corresponde al hablante.

Segunda. Corresponde al oyente

Tercera. Corresponde a la persona que no actúa ni como hablante ni como oyente.

En este sentido, y en lo que se refiere a la adquisición del lenguaje y al desarrollo de la comunicación infantil, son aspectos básicos, o aún más, imprescindibles para la realización del propio proceso comunicativo, dado que sitúan a los participantes en el contex to de habla. Esta necesidad implicaría una rápida adquisición de los mismos por parte de los niños. Sin embargo, lo que parece dificultar su dominio se encuentra en relación con el hecho de que, mientras los sustantivos hacen referencia al mismo objeto o sujeto, los pronombres no se identifican con uno determinado. Aunque su significado no varía su referente es cambiante («yo» = primera persona, independientemente de quien sea el que habla). Son signos vacíos hasta que los hablantes los asumen y no existen, como en el caso de la primera y segunda persona, más que actualizados en el discurso (Benveniste, 1970). Asimismo Bühler (1968) señala que la utilización de estos términos va desde el primitivo uso deíctico (señalamiento en el espacio) hasta la referencia anafórica dentro del discurso. Por tanto, los pronombres desempeñan un papel primordial en la comunicación, y sobre todo en el diálogo. Diversos autores han dedicado una atención especial a este tema, dado su interés para el estudio de la adquisición del lenguaje, que incluye además otros desarrollo implicados en ella.

E.V. Clark y sus colaboradores (1978a, 1978b), plantean a partir de estudios experimentales y desde un punto de vista semántico, que la adquisición de estos términos tiene lugar en función de la complejidad de los rasgos semánticos que incluyen. Los más simples son «yo» y «tú» — siendo los primeros que se adquieren-y posteriormente «él». Esto es, los niños elaboran hipótesis sobre las funciones de los pronombres, e identifican «yo» y «tú» con roles conversa- 
cionales como único rasgo semántico. En este sentido, «yo» correspondería al hablante y «tú» al oyente. Consecuentemente para este autor los errores de inversión que aparecen en algunos enunciados infantiles se explican en función de la formulación de hipótesis erróneas que consistirían en la identificación de los pronombres con las personas («yo»= adulto $\mathrm{y}$ «tú»= niño).

Por otor lado, R. Charney (1980), desde una perspectiva funcional, afirma que la adquisición de estos términos tiene lugar en la comunicación interpersonal. Esto es, el niño aprende aquellos pronombres que son importantes para él en los roles que desempeña (en el de hablante «yo», y en el de oyente «tú») y, sólo posteriormente, será capaz de abstraer la noción de su rol en la conversación, coincidiendo con el uso que de ellos hacen los adultos.

S. Chiat (1981a, 1981b), desde esta misma orientación, matizó algunos aspectos, señalando la necesidad de que el niño sea capaz de discriminar las estructuras lingüísticas, que codifican los mensajes expresando acciones de objetos o acciones sobre estos objetos, identificando el contenido de las mismas. Así, la adquisición de los pronombres resultaría de la confrontación de formas singulares en contextos particulares, abordando características co-ocurrentes de forma y contenido simultáneamente. Asimismo, generalizan las características extralingüísticas, semánticas y formales, a otros contextos, que son también limitados y semejantes, consiguiendo separar la forma pronominal de la estructura lingüística, identificándola con el rol de habla. Una de las características más sobresalientes para la mayoría de los niños sería precisamente el rol expresado, lo cual explicaría la baja frecuencia de errores de inversión en la producción infantil.

Todas estas consideraciones nos llevan a plantear el presente trabajo desde una perspectiva funcional, con el fin de observar la adquisición y desarrollo pronominal en niños cuya lengua materna es el español, así como las implicaciones que puedan tener esta evolución en otros aspectos del desarrollo infantil. Teniendo en cuenta, además, que apenas existen estudios en nuestro idioma sobre este tema, y dada la especificidad del paradigma pronominal en lengua española frente al inglés, se hacen necesarios estudios de este tipo.

\section{ASPECTOS METODOLOGICOS}

La forma más adecuada de llevar a cabo el estudio de estos términos, dada la corta edad de los sujetos, es el análisis de las formas pronominales en la producción verbal espontánea de estos niños por medio de la observación natural y a través de un estudio longitudinal. Como señala Miller (1978), el instrumento más eficaz para llegar a conocer la naturaleza compleja de los aspectos de la adquisición del lenguaje, es precisamente, la cuidadosa observación de la conducta verbal infantil. Por otro lado, y en esta misma línea, Baltes y Nesselroade (1981) afirman que tan sólo a través de los estudios longitudinales se pueden descubrir los cambios evolutivos. Por lo tanto, la metodología longitudinal junto con la observación natural se nos ofrece como la más adecuada a nuestro estudio.

Además, hemos observado una contradicción entre los resultados obtenidos a partir de estudios experimentales sobre este tema (Clark, y colaboradores, 1978a, 1978b), frente a los que utilizan una metodología de observación natural (Nelson, K., 1973; De Villiers y De VIlliers, 1974). Esto es, los primeros, cen- 
trados fundamentalmente en el aspecto comprensivo de estos términos, afirman que los niños no son capaces de utilizarlos correctamente hasta una edad avanzada con respecto a la adquisición del lenguaje; mientras que los segundos, centrados en la producción lingüística, encuentran que dichos términos aparecen en las primeras expresiones verbales infantiles.

Así pues, se nos planea la necesidad de estudiar las pautas de adquisición y desarrollo de los pronombres, tanto en el aspecto funcional como en el de la evolución lingüística; esto es, como se produce el paso de la utilización meramente deíctica al uso lingüístico propiamente dicho de estos términos. Todo ello requiere una observación exhaustiva, tanto con respecto al momento en que se produce su aparición en la producción verbal infantil, como de los cambios que tienen lugar en el curso de su adquisición y evolución, bien en las formas de uso, bien en la expresión lingüística propiamente dicha.

\section{Sujetos}

En el presente trabajo se estudió la produccción verbal de tres niños, de procedencia ambiental diferente, desde que contaban dos años de edad hasta que cumpieron tres. Carlos y Celia provienen de un medio familiar en el que no se observarı problemas psicosociales ni afectivos aparentes; mientras que $\mathrm{Va}$ nesa vive en una institución para menores protegidos. Estos medios conllevan diferencias en cuanto a los niveles de desarrollo lingüístico (sintáctico, semántico y pragmático), como ya ha sido puesto de manifiesto en estudios anteriores (G. Shum, 1988), así como diferencias en la adquisición y uso de los términos deícticos (Shum y otros, 1989).

\section{Procedimiento}

\section{Recogida de los Datos}

Los datos fueron recogidos, a través de observación natural, mediante video. Para ello se realizaron registro de treinta minutos de duración de la producción verbal infantil, con una periodicidad mensual. La observación consistía en la grabación de una situación no estructurada de juego en la que los niños interactuaban con adultos familiares y habituales. Los juguetes utilizados eran similares para todos los niños y propios de su edad: cuentos, construcciones, coches, etc. Posteriormente se transcribieron los mensajes emitidos por el adulto y le niño, respetando el turno de emisión de los mismos, así como la descrición de la situación contextual para la mejor comprensión de los mensajes.

\section{Análisis de los Datos:}

Una vez realizada la transcripción de la conversación adulto-niño, fueron analizados todos los enunciados infantiles que incluían pronombres personales en cualqueira de sus formas (Ver cuadro I). Posteriormente, se analizaron cada una de las categorías, observando su momento de aparición, así como las formas de uso en función de determinadas características pronominales. Este análisis se realizó en los niveles sintáctico, semántico y pragmático, teniendo en cuenta las consideraciones más importantes con respecto a la evolución de las formas pronominales, recogidas en la literatura sobre la adquisición del lenguaje, mencionadas anteriormente. 
CuAdro I

Categorias del pronombre personal utilizadas para el análisis

\begin{tabular}{|c|c|c|c|c|c|}
\hline & & \multirow{2}{*}{\multicolumn{2}{|c|}{$\begin{array}{c}\text { SIN CASO } \\
\text { (Formas tónicas) }\end{array}$}} & \multicolumn{2}{|c|}{$\begin{array}{c}\text { CON CASO } \\
\text { (Formas átonas) } \\
\text { COMPLEMENTO }\end{array}$} \\
\hline & & & & & INDIRECTO \\
\hline \multicolumn{2}{|c|}{ SINGULAR } & \multicolumn{2}{|c|}{ yo, mí, conmigo } & \multicolumn{2}{|c|}{ me } \\
\hline \multirow{2}{*}{\multicolumn{2}{|c|}{ PLURAL }} & Masc. & nosotros & \multirow{2}{*}{\multicolumn{2}{|c|}{ nos }} \\
\hline & & Fem. & nosotras & & \\
\hline \multicolumn{2}{|c|}{ SINGULAR } & \multicolumn{2}{|c|}{ tú, tí, contigo } & \multicolumn{2}{|c|}{ te } \\
\hline \multirow{2}{*}{\multicolumn{2}{|c|}{ PLURAL }} & Masc. & vosotros & \multirow{2}{*}{\multicolumn{2}{|c|}{ os }} \\
\hline & & Fem. & vosotras & & \\
\hline \multirow[t]{5}{*}{ NO } & \multirow{3}{*}{ SING. } & Masc. & él & lo & \multirow{3}{*}{ le } \\
\hline & & Fem. & ella & la & \\
\hline & & Neut. & ello & lo & \\
\hline & \multirow{2}{*}{ PL. } & Masc. & ellos & los & \multirow{2}{*}{ les } \\
\hline & & Fem. & ellas & las & \\
\hline \multicolumn{2}{|c|}{ REFLEXIVO } & \multicolumn{2}{|c|}{ sí, consigo } & \multicolumn{2}{|c|}{ se } \\
\hline
\end{tabular}

A. La primera diferenciación que realizamos en el análisis de datos, está relacionada con la variación de caso, característitica propia del pronombre en lengua española. Así agrupamos los términos pronominales en:

- Formas que no ofrecen contenido causal, fonéticamente tónicas, que no llevan en sí mismas la indicación de su función en el enunciado.

- Formas con contenido casual, fonéticamente atónas, que indican la función que cumplen en el enunciado; se caracterizan por las posibilidades combinatorias que tienen en la oración, entre sí o con otros elementos de la misma, añadiéndose a la palabra anterior (proclítico) o posterior (enclítico).

Esta diferenciación nos permite diferenciar aquellos usos pronominales fruto de la imitación y memorización infantil, de aquellos en los que existe una mayor elaboración de los enunciados por parte de los niños. Dadas sus características, las formas átonas dependen en mayor medida de las estructuras lingüís- 
ticas que las tónicas, siendo su variabilidad mayor en cuanto a funciones oracionales.

Posteriormente, y a partir de esta diferenciación sintáctica, analizamos cada uno de los tipos:

\section{Formas tónicas}

Se tuvo en cuenta el momento de aparición de los pronombres que corresponden a esta categoría, así como su frecuencia de uso. Al mismo tiempo, se analizó a nivel cualitativo la transformación sintáctica de los enunciados infantiles (en función de la mayor o menor conplejización de éstos) a lo largo de todo el estudio. De esta forma podemos determinar, en cierto sentido, la transformación del uso de estos pronombres desde el mero señalamiento (deíctico) a formas más lingüísticas. Este análisis se realizó teniendo en cuenta las funciones pragmáticas (informativa o demanda) que cumplen estos enunciados, así como la función sintáctica que desempeñan los pronombres incluidos en ellos (Sujeto o complementos).

A nivel morfológico se tuvo en cuenta el género de las formas pronominales (femenino, masculino), el número de las mismas (singular, plural), y en especial la persona a la que hacen referencia (primera, segunda o tercera). Esta última diferenciación es importante para nuestro estudio no sólo por tratarse de una característica del paradigma pronominal, sino porque además nos permite observar la identificación de los pronombres personales y los roles correspondientes en la conversación.

\section{Formas átonas:}

A nivel pragmático fue analizada la función que cumplen los enunciados en los que aparecen las formas pronominales (informativa o demanda). Posteriormente se categorizaron en relación a la función sintáctica que desempeñan en la oración (complemento directo o indirecto). Esto es especialmente importante en la función de demanda, en la que se tuvo en cuenta el tipo de construcción sintáctica para estudiar su variedad y evolución, ya que esto nos permite observar el nivel de conocimiento social que posee el niño, reflejado en la formulación de sus enunciados. Así, observamos el lugar que ocupa el pronombre con respecto al verbo (anterior o posterior), y el modo verbal en que se expresa éste (indicativo, imperativo). Dentro de cada una de estas categorías, al igual que en el análisis de las formas tónicas, se tuvo en cuenta el momento de aparición de estos términos, y su frecuencia de uso.

A.) nivel semántico se analizó la variedad de los verbos, es decir, cuántos verbos distintos utilizan los niños con estos pronombres. Así como también, la reflexividad o no de los mismos, con objeto de observar las diferencias en cuanto a la predicación de los enunciados infantiles. La mayor variedad verbal implica una mayor riqueza semántica, ya que a medida que evoluciona el lenguaje en el niño se complejiza la estructura lingüística, lo cual exige un mayor número de verbos. Por otro lado, las características de los verbos reflexivos implican necesariamente la presencia del pronombre personal en los enunciados, lo cual supone una automatización en el uso de éstos.

Por último, dentro de las formas átonas, y respecto a los pronombres de tercera persona «la», «le» y «lo», analizamos si su uso es deíctico o anafórico, dado que se trata de las únicas en las que se puede evidenciar de forma clara. Consi- 
deramos estos términos como deícticos cuando los utilizan como recursos comunicativos, y como anafóricos cuando tienen una dimensión más lingüística, esto es, cuando asumen el significado de una palabra anteriormente mencionada en el discurso, bien del adulto o del propio niño.

B.) Se analizaron también, cualitativamente, los usos inadecuados que los niños hacen de estos términos, con respecto a ambos grupos de pronombres personales. Este análisis nos pareció interesante, puesto que nos permite investigar el nivel de conocimiento infantil sobre el uso adecuado de estos términos en el diálogo. Al mismo tiempo, nos permiten formular hipótesis con el objetivo de investigar estrategias o procesos que subyacen a los aprendizajes lingüísticos de los niños. Para ello se observó:

- Los errores en la identificación de los pronombres con el rol de los participantes en el habla, es decir, si existen inversiones.

- Las omisiones: la no aparición de las formas pronominales en aquellos enunciados en los que lingüísticamente son necesarias, cambiando, de este modo, el contenido de los mismos. Este error supone un desconocimiento de los usos de los pronombres personales por parte de los niños, constituyendo, por lo tanto, el error más grave.

- La adición, esto es, la utilización de los pronombres cuando no son necesarios porque su contenido está ya explicitado por un sintagma nominal o por otra forma pronominal. También se tuvo en cuenta cuando esta adición altera la estructura sintáctica, convirtiéndola en erronea.

- La concordancia (género, número o persona), bien con respecto al verbo (concordancia sintáctica), bien respecto a los objetos o sujetos a los que se refiere afectando al contenido.

\section{ANALISIS DE LOS PRONOMBRES PERSONALES}

\section{Formas tónicas}

Los pronombres personales en sus formas tónicas aparecen en la producción lingüística de estos niños desde un primer momento, aunque, con diferencias respecto a su frecuencia de uso. Las formas «yo - tú» aparecen al mismo tiempo, pero con un mayor predominio del pronombre «yo» (ver Tabla I). Su uso es adecuado, ya que en la producción lingüística de estos niños no se observan errores de inversión; esto es, la forma pronominal «yo» es utilizada como referencia al sujeto-hablante, y la forma «tú » al oyente dentro de los contextos conversacionales. La frecuencia de uso de estos términos está relacionada con los procesos comunicativos, dado que en la lengua española, salvo en algunas excepciones, no es obligatorio el uso del pronombre personal porque el verbo incluye marcadores de persona. De esta manera encontramos que, a esta edad, «yo» se usa fundamentalmente para enfatizar el sujeto de la acción. [Ejs.: (1), (2) y (3)]

(1) Yo doy un salto/ quiero una silla/ esa. Celia, $(2 ; 0)$

(2) A: ¿Disparamos? (jugando con una escopeta)

$\mathrm{N}$ : Yo voy a disparar/ yo voy a disparar

Vanesa $(2 ; 11)$

(3) A: ¿Qué has hecho con la tierra?

$\mathrm{N}$ : Yo he hecho montañas así.

Carlos $(2 ; 4)$

Al mismo tiempo aparecen en enunciados en los que el niño se opone a las 


\section{4}

TABLA I

Las formas tónicas en la producción lingüística de los niños. (Frec. absolutas)

CARLOS

\begin{tabular}{|llccccc|}
\hline Edad & Yo & Tu & El/Ella & Mi & Ti & Plurales \\
\hline $2 ; 0$ & 1 & 1 & -- & -- & -- & -- \\
$2 ; 1$ & 5 & 2 & -- & -- & -- & -- \\
$2 ; 3$ & 18 & 9 & -- & 1 & -- & -- \\
$2 ; 4$ & 8 & 1 & -- & -- & -- & -- \\
$2 ; 5$ & 12 & -- & -- & 1 & 1 & -- \\
$2 ; 6$ & 10 & 1 & -- & -- & -- & -- \\
$2 ; 7$ & 8 & 3 & -- & -- & -- & -- \\
$2 ; 8$ & 6 & 2 & -- & 1 & -- & 1 \\
$2 ; 9$ & 7 & -- & -- & -- & -- & 1 \\
$2 ; 10$ & 9 & 9 & -- & -- & -- & 3 \\
$2 ; 11$ & 10 & 5 & 2 & 2 & -- & 1 \\
\hline
\end{tabular}

\section{CELIA}

\begin{tabular}{|lcccccc|}
\hline Edad & Yo & Tu & El/Ella & Mi & Ti & Plurales \\
\hline $2 ; 0$ & 2 & 2 & -- & -- & -- & -- \\
$2 ; 1$ & 3 & 3 & -- & -- & -- & -- \\
$2 ; 3$ & 6 & 2 & -- & -- & -- & -- \\
$2 ; 4$ & 6 & 1 & -- & -- & -- & -- \\
$2 ; 5$ & 7 & 2 & -- & -- & -- & -- \\
$2 ; 6$ & 7 & 3 & -- & -- & -- & -- \\
$2 ; 8$ & 15 & -- & -- & 2 & -- & -- \\
$2 ; 9$ & 12 & 1 & -- & -- & -- & -- \\
$2 ; 10$ & 10 & -- & 2 & -- & -- & -- \\
$2 ; 11$ & 4 & 3 & -- & -- & -- & 1 \\
$3 ; 0$ & 10 & 3 & 1 & -- & -- & -- \\
\hline
\end{tabular}

VANESA

\begin{tabular}{|lcccccc|}
\hline Edad & Yo & Tu & El/Ella & Mi & Ti & Plurales \\
\hline $2 ; 3$ & -- & -- & -- & -- & 1 & -- \\
$2 ; 4$ & 2 & 4 & 1 & -- & -- & -- \\
$2 ; 5$ & 5 & 8 & -- & 2 & 6 & -- \\
$2 ; 6$ & 1 & -- & -- & -- & 1 & -- \\
$2 ; 7$ & -- & 6 & -- & -- & -- & -- \\
$2 ; 11$ & 7 & 1 & -- & 1 & -- & -- \\
$3 ; 0$ & 6 & -- & -- & -- & -- & -- \\
\hline
\end{tabular}


demandas, propuestas o informaciones del adulto, lo cual es también una forma de énfasis, pero con características pragmáticas distintas. (Ejs.: (4), (5) y (6).

(4) A: ¿Te ayuda mamá un poco?/ vamos a ver. (juego de construcción)

N: Yo/ yo quiero.

(5) A: ¿Es un muñeco?/ no creo.

$\mathrm{N}$ : Yo si creo

Celia $(2 ; 5)$

(6) A: Te lo doy yo ¿eh?

$\mathrm{N}: \mathrm{No} /$ yo

Vanesa $(2 ; 5)$

Estas formas pragmáticas aparecen en todos los niños. Las diferencias entre ellos se refieren a la distinta distribución de las frecuencias de uso, que están más relacionadas con sus características individuales que con el propio proceso de adquisición lingüístico.

En lo que sí aparecen marcadas diferencias, entre los niños de medio familiar y la niña de medio institucional, es en lo referente a la estructura gramatical de los mensajes que incluyen estos pronombres. Carlos y Celia producen enunciados cuya complejidad estructural aumenta progresivamente a medida que avanzan en edad; de esta manera, estos términos forman parte de enunciados más elaborados linguísticamente, lo cual supone una evolución en la adquisición pronominal. Es decir, estos niños son capaces de usar el pronombre de primera persona como sujeto de una acción expresada verbalmente, [Ejs.: (7), (8)] mientras que Vanesa hasta los tres años utiliza, casi de forma exclusiva, el pronombre «yo» (sólo, o acompañado de un adverbio), como función de indicación, esto es, de forma eminenentemente deíctica [Ejs.: (9), (10)].

(7) N: Pero yo juego sólo con mis amigos en mi clase.

(8) N: Yo le doy a comer algo.

(9) A: Ahora voy en este coche

$\mathrm{N}$ : Yo allí

Carlos $(2 ; 11)$

Celia $(3 ; 0)$

Vanesa $(2 ; 5)$

(10) N: Se val y este coche llevo yo.

Vanesa $(3 ; 0)$.

El modo en que utilizan el pronombre «tú» es muy similar al de la primera persona, sin embargo, dadas las características de este término, predomina su uso como forma de demanda. El niño requiere de su interlocutor la realización de acciones a través de expresiones que incluyen este pronombre. La producción lingüística de esta función se desarrolla desde la forma más primitiva, que consiste en la verbalización del pronombre como único elemento del enunciado (forma habital para Vanesa) [Ej.: (11)], hata producciones más complejas en las que se expresan las acciones a realizar. Dentro de este tipo, frecuentemente en los niños de medio familiar, se observan dos formas generales de estructuración de los enunciados: «pronombre + verbo en indicativo» [Ej.: (12)], y «verbo en imperativo + pronombre» $[\mathrm{Ej} .:$ (13)].

(11) A: Mira te sientas ahí.

N: Y tú ahí.

Vanesa $(2 ; 5)$

(12) A: Ponte aquí/ así al mismo lado.

$\mathrm{N}$ : Entonces tú te pones aquí.

Carlos $(2 ; 4)$

Celia $(3 ; 0)$.

(13) N: Déjame hacer mis deberes

El pronombre de tercera persona «él», y las formas plurales aparecen esporádicamente, y rara vez desempeñando la función de sujeto. Suelen utilizarlas acompañadas de preposición con función de complementos oracionales. En los 
enunciados de Carlos y Celia encontramos esta forma de uso de los pronombres incluidos en enunciados gramaticalmente complejos [ $\mathrm{Ej} .:$ (14)], mientras que en los de Vanesa aparecen únicamente las formas tónicas «mí» «tí» acompañadas de preposición en enunciados sin verbo explícito, lo cual constituye un modo de expresión propio de los primeros momentos del desarrollo del lenguaje infantil [Ej.: (15)].

(14) N: Yo juego con ellos en la guardería cuando me vengan a buscar

Carlos $(2 ; 11)$

(15) A: Un poquito ¿eh?. (repartiendo azúcar)

$\mathrm{N}: A m i$.

Vanesa $(2 ; 5)$

En resumen, podemos decir que, la secuenciación en la adquisición de los pronombres personales en su forma nominativa, se debe, por una parte, a la propia complejidad de estos términos. En este sentido los pronombres «yo» «tú» son formas más sencillas que «él» y los plurales. Estos últimos poseen un mayor número de componentes, que implicitan más diversidad en las relaciones, esto es, mayor número de personas con diferentes relaciones, y más variabilidad en los referentes, (ej.: «nosotros = yo + tú, yo + él, yo + tú + él). Por otro lado, y fundamentalmente, dicha secuenciación se debe a que «yo» y «tú» son componentes imprescindibles para la comunicación. Es decir, el niño está inmerso en una situación de interacción didáctica desde su nacimiento, en la cual el uso de estos pronombres es, evidentemente, necesario. El hecho de que no se detecten errores de inversiones de roles ni aún en los primeros momentos de este estudio, nos hace suponer que el desarrollo de estos términos está muy relacionada con la adquisición y consolidación de los roles conversacionales en la etapa preverbal.

\section{Las formas átonas: «me» - «te» - «se»}

A través de los análisis observamos que los pronombres personales «me»y «te» son usados por Celia y Carlos desde el comienzo del estudio (2;0 años), aunque en la producción verbal de Celia existe una mayor regularidad en lo que se refiere a la utilización de estos pronombres, mientras que en el caso de Carlos su uso se regulariza en torno a los dos años y cinco meses. (Ver Tabla II).

Puede considerarse que los enunciados verbales en los que aparecen estos pronombres, son prácticamente semejantes en su forma gramatical a los del lenguaje adulto. Esto es, son utilizados en mensajes informativos con estructuras más o menos complejas, donde «me» y «te» se presentan bien como objeto directo o como objeto indirecto. En el caso acusativo aparecen fundamentalmente en enunciados con verbos reflexivos, los cuales conllevan necesariamente la presencia de tales pronombres [Ejs.: (16), (17)]. Por otro lado, la expresión de estos términos en la comunicación diádica es correcta, lo cual nos hace suponer que su adquisición está muy relacionada con el uso de los verbos pronominales. Con el resto de los verbos en los que los términos «me» y «te» aparecen en función de objeto indirecto, excepto en casos como «gustar», «querer», «apetecer», etc. cuyo uso exige el acompañamiento de estos pronombres, se refleja de una manera más clara la adecuada o inadecuada utilización de estas formas gramaticales [Ej.: (18)]. en líneas generales podemos decir, que el uso de los mismos es correcto en el proceso comunicativo. Por ello, Celia y Carlos conocen tanto el rol de hablante como el de oyente, no sólo como sujetos sino también como recep- 
TABLA II

Las formas átonas me, te, se, en la producción lingüística de los niños. (Fre. abs.).

CARLOS

\begin{tabular}{|c|c|c|c|c|c|}
\hline \multicolumn{3}{|c|}{ INFORMATIVA } & \multicolumn{3}{|c|}{ DEMANDA } \\
\hline \multirow{2}{*}{$\begin{array}{l}\text { Dativo } \\
\text { EDAD }\end{array}$} & \multirow{2}{*}{\multicolumn{2}{|c|}{$\begin{array}{l}\text { ACUSATIVO } \\
\text { Reflex }\end{array}$}} & \multirow{2}{*}{$\begin{array}{l}\text { V. (IMP) } \\
\text { NO-REF. }\end{array}$} & \multicolumn{2}{|c|}{ PRON. + } \\
\hline & & & & + PRON & V. (IND) \\
\hline $2 ; 0$ & 1 & 4 & -- & -- & -- \\
\hline $2 ; 1$ & 4 & 2 & -- & -- & -- \\
\hline $2 ; 3$ & 14 & 6 & -- & -- & -- \\
\hline $2 ; 4$ & 7 & 8 & -- & 1 & 1 \\
\hline $2 ; 5$ & 1 & 32 & -- & 4 & -- \\
\hline $2 ; 6$ & 20 & 22 & -- & -- & -- \\
\hline $2 ; 7$ & 10 & 10 & -- & -- & 3 \\
\hline $2 ; 8$ & 20 & 25 & -- & 2 & -- \\
\hline $2 ; 9$ & 13 & 38 & -- & 1 & -- \\
\hline $2 ; 10$ & 8 & 18 & -- & 4 & 2 \\
\hline $2 ; 11$ & 4 & 15 & -- & -- & -- \\
\hline
\end{tabular}

\section{CELIA}

\begin{tabular}{|c|c|c|c|c|c|}
\hline \multicolumn{3}{|c|}{ INFORMATIVA } & \multicolumn{3}{|c|}{ DEMANDA } \\
\hline Dativo & & IVO & V. (IMP) & & RON. + \\
\hline EDAD & & Reflex & NO-REF. & + PRON & V. (IND) \\
\hline $2 ; 0$ & 7 & 10 & -- & 3 & -- \\
\hline $2 ; 1$ & 3 & 10 & 2 & 4 & 1 \\
\hline $2 ; 3$ & 5 & 2 & -- & 11 & 1 \\
\hline $2 ; 4$ & 10 & 15 & -- & 2 & 3 \\
\hline $2 ; 5$ & 11 & 4 & -- & 6 & 3 \\
\hline $2 ; 6$ & 4 & 11 & 5 & 4 & 2 \\
\hline $2 ; 8$ & 12 & 7 & -- & 2 & -- \\
\hline $2 ; 9$ & 3 & 3 & -- & 5 & -- \\
\hline $2 ; 10$ & 6 & 7 & 1 & -- & -- \\
\hline $2 ; 11$ & 4 & 4 & -- & 1 & 1 \\
\hline $3 ; 0$ & 7 & 17 & -- & 15 & 4 \\
\hline
\end{tabular}

\section{VANESA}

\begin{tabular}{|c|c|c|c|c|c|}
\hline & MATI & & & EMANDA & \\
\hline Dativo & & IIVO & V. (IMP) & & RON. + \\
\hline EDAD & & Reflex & NO-REF. & + PRON & V. (IND) \\
\hline $2 ; 3$ & 1 & -- & -- & -- & -- \\
\hline $2 ; 4$ & -- & -- & -- & 1 & -- \\
\hline $2 ; 5$ & -- & -- & -- & 1 & -- \\
\hline $2 ; 6$ & 2 & 8 & -- & 1 & -- \\
\hline $2 ; 7$ & 4 & 8 & -- & -- & -- \\
\hline $2 ; 11$ & 4 & 10 & -- & 2 & -- \\
\hline $3 ; 0$ & 1 & 31 & -- & 2 & -- \\
\hline
\end{tabular}


tores directos o indirectos de la acción verbal (funciones habituales de «me» y «te»); utilizando «me» para sí mismos y «te» para su interlocutor. Sin embargo, aparecen algunas inadecuaciones en el uso lingüístico que, debido a su interés, serán comentados posteriormente.

(16) N: Pero $m e$ voy a mi casa

(17) N: ¿Quieres que me vaya? si o no.

(18) N: Por eso me deja todos los días su tractor.

Carlos $(2 ; 10)$

Celia $(3 ; 0)$

CArlos $(2 ; 10)$

Respecto a la función de demanda, que aparece con menor frecuencia, encontramos dos tipos de estructuras:

1. Verbo imperativo + pronombre (+ complementos) [Ej.: (19)]

2. Pronombre + verbo indicativo (+ complementos) [Ej.: (20)]

La primera de ellas es más frecuente en la producción verbal de ambos niños. En un primer momento, es utilizada con verbos muy usuales en su vida cotidiana, relaciones con las actividades de juego: «bajar», «tirar», «bañar», «dar», «caer», «esconder», etc. A medida que se van complejizando los juegos, aumenta de forma progresiva la variedad de los verbos, que además van haciendo menor referencia a las acciones contextuales.

Respecto al segundo tipo encontramos una menor frecuencia, dado que se trata de una forma más indirecta de demanda y exige un nivel más elevado de desarrollo de conocimiento, no sólo lingüístico sino también social y físico (Shum y otros, 1989).

(19) A: Se te cae el tractor

N: Déjamelo

Celia $(3 ; 0)$

(20) N: Quiero que me dejes porque si no, me voy a enfadar contigo.

Celia $(3 ; 0)$

La niña perteneciente al medio institucional presenta cierto retraso en el uso de estos pronombres. Hasta casi los dos años y ocho meses no aparecen apenas «me» y «te» en la producción verbal de Vanesa. La estructuración de dichos enunciados es mucho más simple que la de los niños de medio familiar. En la función informativa predomina como en los otros niños, el uso de estos términos deícticos como objeto directo con verbos reflexivos [Ej.: (21)]. Su utilización, aunque más escasa, también es correcta. El caso del objeto indirecto es todavía menos frecuente, se observa una menor variedad de verbos y no muestra una evolución evidente en sus enunciados a lo largo del estudio. Generalamente no produce apenas errores lingüísticos en estos términos dado que sus enunciados suelen ser repetitivos y estereotipados.

En lo que a la función de demanda se refiere sólo aparece la forma:

- Verbo imperativo + Pronombre. [(Ej.: (22)]

Su uso es esporádico y utiliza siempre el mismo tipo de verbos. Además, este se produce a una edad avanzada con respecto al período de estudio $(3 ; 0$ años). Antes de este momento los tipos de demanda que incluyen estos términos son, como ya se ha comentado, las formas pronominales «a mí», "para mí», etc.

\section{(21) N: Me voy corriendo}

(22) N: Dame eso/ dame eso. (Un barco)

Vanesa $(3 ; 0)$

Vanesa $(2 ; 11)$

Por otro lado, respecto al conocimiento de los roles conversacionales que implica el uso de estos términos, podemos señalar que no se observan inversiones, ni confusiones en cuanto a la persona hablante y oyente. 
El pronombre de tercera persona «se» aparece en los enunciados producidos por estos niños fundamentalmente acompañado a verbos reflexivos, y es utilizado de forma gramaticalmente adecuada [Ejs.: (23), (24)]. Al igual que en los pronombres anteriores la niña de medio institucional presenta una menor variedad en el uso de verbos, así como en lo que respecta al uso de los pronombres. Esto es, mientras Celia y Carlos utilizan con este tipo de verbos las formas pronominales de primera, segunda y tercera persona, Vanesa principalmente utiliza la forma «se», correspondiendo sus enunciados con frases hechas utilizadas habitualmente en la comunidad hablante [Ej.: (25)].

(23) N: Se ha ido mi nana a Vallecas

Celia $(2 ; 4)$

(24) A: No hombre, no se ha roto

N: Si mira/ mira como si se ha roto.

Carlos $(2 ; 10)$

(25) N: No se abre la puerta.

Vanesa $(3 ; 0)$

Por otra parte este pronombre, «se», también es utilizado en enunciados en los que aparece como indicador de impersonalidad o de voz pasiva, sin embargo, en tales casos dicho pronombre ha perdido su carácter reflexivo y pronominal, de ahí que no aparezca en nuestro análisis.

\section{Formas átonas: «la» - «lo» - «le».}

Carlos y Celia utilizan este tipo de pronombres desde el comienzo de este estudio ( $2 ; 0$ años), aunque se observa un aumento en la frecuencia de uso a partir de los dos años y tres meses. Estos términos aparecen tanto en los enunciados de tipo informativo, como en aquellos que cumplen una función de demanda. En estos últimos la frecuencia es menor, dado que en la producción verbal infantil también lo es el número de enunciados dentro de esta categoría pragmática con respecto a las enunciativas, (Shum, 1986) (Ver Tala III). En la producción linguística de estos niños de observa, tanto en la función informativa como en la de demanda, una mayor frecuencia de las formas «la» y «lo», lo cual puede deberse a los tipos de verbos utilizados, que son en su mayoría transitivos.

Respecto a las características anafóricas y deíctica propia de estos pronombes, aparecen las dos desde el comienzo del estudio ( $2 ; 0$ años), el uso de una $\mathrm{u}$ otra está ligado a las características del enunciado y del contexto comunicativo [Ejs.: (26), (27), (28)].

(26) A: ¿Y Diego que va a llevar?

$\mathrm{N}$ : No/ porque yo le voy a dejar este coche/ pero cuando me vaya me lo da el rojo.

Carlos $(2 ; 11)$

(27) A: Voy a dar la tortilla a la niña

N: No se la des.

(28) A: ¿Por qué no tienes hermanito?

$\mathrm{N}$ : Porque no le ha nacido a mamá.

Celia $(3 ; 0)$

Celia $(3 ; 0)$

En el caso de Vanesa, el uso de los pronombres «la», «lo»y «le», es más tardío, puede decirse que comienza hacia los dos años y seis meses. Utiliza dichos términos tanto en enunciados cuya función es informativa como en los de demanda, y al igual que los otros niños, son más habituales en la informativa. Las diferencias fundamentales que presenta esta niña con respecto a los otros estriban en que la frecuencia de uso de estos pronombres es mucho menor y la forma de utilización es fundamentalmente deíctica [Ej.: (29), (30)]. En el ca- 


\section{0}

TABLA III

Las formas átonas: la, lo, le, en la producción lingüistica de los niños. (Frec. Abs.)

CARLOS

\begin{tabular}{|c|c|c|c|c|c|c|c|c|}
\hline \multicolumn{4}{|c|}{\begin{tabular}{|l} 
INFORMATIVA \\
\end{tabular}} & \multicolumn{5}{|c|}{ DEMANDA } \\
\hline \multirow{2}{*}{$\begin{array}{l}\text { ACUSAT } \\
\text { Edad }\end{array}$} & LA-LO & \multicolumn{2}{|c|}{ DATIVO. LE } & \multicolumn{3}{|c|}{ ACUSA. LA-LO } & \multicolumn{2}{|c|}{ DATIVO. LE } \\
\hline & Anaf. & Deic. & Anaf. & Deic. & Anaf. & Deif. & Anaf. & Deic. \\
\hline $2 ; 0$ & 1 & 1 & -- & -- & $-\ldots$ & - - & -- & -- \\
\hline $2 ; 1$ & 2 & 2 & -- & -- & -- & 15 & -- & -- \\
\hline $2 ; 3$ & 6 & 2 & - & 2 & -- & -- & -- & -- \\
\hline $2 ; 4$ & 5 & 3 & -- & -- & - & 1 & -- & -- \\
\hline $2 ; 5$ & 6 & 3 & 1 & 2 & -- & 2 & -- & -- \\
\hline $2 ; 6$ & 10 & 2 & -- & - & - - & -- & -- & -- \\
\hline $2 ; 7$ & 4 & 1 & 1 & -- & -- & 1 & -- & -- \\
\hline $2 ; 8$ & 1 & 3 & 2 & 1 & 1 & -- & -- & -- \\
\hline $2 ; 9$ & 15 & -- & 2 & 3 & -- & -- & -- & - \\
\hline $2 ; 10$ & 15 & 7 & 4 & -- & 1 & 3 & -- & -- \\
\hline $2: 11$ & 3 & 8 & 2 & 2 & -- & -- & -- & -- \\
\hline
\end{tabular}

CELIA

\begin{tabular}{|c|c|c|c|c|c|c|c|c|}
\hline & ORMA & IIVA & & & & EMAN & & \\
\hline ACUSAT & & DATIV & LE & $A C L$ & A. LA. & & DATIVC & LE \\
\hline Edad & Anaf. & Deic. & Anaf. & Deic. & Anaf. & Deif. & Anaf. & Deic. \\
\hline $2 ; 0$ & 1 & -- & -- & -- & -- & 12 & 2 & -- \\
\hline $2 ; 1$ & -- & -- & -- & -- & 3 & -- & 4 & -- \\
\hline $2 ; 3$ & 2 & 3 & 4 & -- & 5 & 3 & 1 & 6 \\
\hline $2 ; 4$ & 4 & 4 & -- & 2 & 1 & -- & -- & -- \\
\hline $2 ; 5$ & 10 & 8 & 1 & -- & 2 & 1 & 1 & 1 \\
\hline $2 ; 6$ & 3 & 5 & 1 & 1 & -- & -- & -- & 1 \\
\hline $2 ; 8$ & 3 & 4 & 1 & 1 & $-\ldots$ & -- & -- & -- \\
\hline $2 ; 9$ & -- & 3 & -- & -- & 1 & -- & -- & -- \\
\hline $2 ; 10$ & 1 & 4 & 1 & 4 & -- & -- & -- & -- \\
\hline $2 ; 11$ & 8 & 7 & 1 & -- & -- & -- & -- & -- \\
\hline $3 ; 0$ & 4 & 11 & 6 & 5 & 2 & -- & -- & 1 \\
\hline
\end{tabular}

VANESA

\begin{tabular}{|c|c|c|c|c|c|c|c|c|}
\hline \multicolumn{4}{|c|}{ INFORMATIVA } & \multicolumn{5}{|c|}{ DEMANDA } \\
\hline \multirow{2}{*}{$\begin{array}{l}\text { ACUSAT. } \\
\text { Edad }\end{array}$} & A-LO & & \multicolumn{3}{|c|}{ ACUSA. LA-LO } & \multicolumn{2}{|c|}{ DATIVO. LE } \\
\hline & Anaf. & Deic. & Anaf. & Deic. & Anaf. & Deif. & Anaf. & Deic. \\
\hline $2 ; 3$ & $-\ldots$ & $-\ldots$ & -- & -- & -- & -- & -- & -- \\
\hline $2 ; 4$ & -- & -- & -- & -- & -- & -- & -- & -- \\
\hline $2 ; 5$ & 2 & - & 3 & - & -- & - & -- & -- \\
\hline $2 ; 6$ & 7 & 7 & 1 & 1 & -- & -- & -- & -- \\
\hline $2 ; 7$ & -- & 3 & -- & 1 & -- & -- & -- & -- \\
\hline $2 ; 11$ & 2 & 2 & 1 & 1 & -- & 1 & -- & -- \\
\hline
\end{tabular}


so de aparecer algunos pronombres con forma anafórica, encontramos que con los mismos términos, y forman parte de enunciados estereotipados y repetitivos.

(29) A: ¿Tiene sueño?

$\mathrm{N}$ : Ahora le doy el tete.

Vanesa $(2 ; 6)$

(3) A: Ahora lo volvemos a poner.

$\mathrm{N}$ : y nos vamos/ ya lo quito

Vanesa $(3 ; 0)$

\section{ANALISIS DE ERRORES}

\section{Formas tónicas}

Como ya se ha comentado anteriormente no se observa en estos errores de inversión con respecto a las personas de estos pronombres (Yo-Tú). Unicamente en el caso de Vanesa encontramos, de forma esporádica - dos veces-, errores con respecto a estas formas pronominales, referentes a la concordancia de la persona con el predicado de la oración.

(31) A: No le gusta la capucha (se refiere a un muñeco)/ lo

ha tirado, ¿No?

$\mathrm{N}$ : Lo ba tirado $t u ́$

Vanesa $(2 ; 5)$

(32) $N$ : La be comprado esa (se refiere a una muñeca)

A: ¿Qué?

$\mathrm{N}$ : Me ha comprado

A: ¿Qué dices?

$\mathrm{N}$ : Me ba comprado esa.

Vanesa $(2 ; 6)$

En el primer ejemplo [(31)], el sujeto aparece en segunda persona y el verbo en primera de singular, como puede observarse el enunciado de la niña exceptuando el sujeto - es una imitación del mensaje del adulto sin trasformar, lo que demuestra cierta limitación con respecto al desarrollo lingüístico de Vanesa.

En el segundo [Ej.: (32)] tampoco concuerdan, el sujeto (tercer persona) y el verbo (primera persona), aunque posteriormente aparece una rectificación por parte de la niña ante la demanda del adulto.

En estas dos secuencias es interesante señalar como Vanesa en el momento en que pasa del uso deíctico de estos pronombres de formas más lingüísticas comete algunos erroes en la producción de sus enunciados.

Aparece también de forma esporádica la utilización de los términos: «mí», «tí», «él» y «vosotros», precedidos de la preposición «de», sustituyendo a las formas pronominales de posesivo. Esto es frecuente en las etapas tempranas del desarrollo de las formas de posesión en los niños; no obstante, y debido a que el análisis de estos pronombres no es objeto de nuestro estudio, no han sido considerados.

\section{Formas átonas: «me» - «te» - «se»}

En lo que se refiere a la utilización de los pronombres «me» «te» «se», se observa la aparición de diversos tipos de errores en función de los niños.

Uno de los errores que aparece con bastante frecuencia en las locuciones de Carlos y Vanea, con verbos que pueden tener forma reflexiva y no reflexiva, es el uso de estos pronombres como reflexivos en aquellos casos en los que no lo son [Ejs.: (33), (34)]. 
(33) N: Yo me bajo por la escalera.

Asimismo, Carlos, utiliza los pronombres personales como reflexivos con verbos que no lo son [Ej.: (35), (36)]. En Vanesa no aparecen estos errores dado que los verbos más usados por esta niña, con pronombres de este tipo, son precisamente los reflexivos.

(35) N: Cuando se tira al suelo se pega un trote/ se tira una montaña grande de arena mojada.

(36) N: En una agua así de grandota se estaba el pato. Carlos $(2 ; 11)$

A través de estos errores se observa como estos niños, que habitualmente utilizan adecuadamente verbos reflexivos con estas formas pronominales, encuentran dificultad al utilizar estos términos deícticos con otros tipos de verbos; así suelen usar los verbos no-reflexivos como si lo fueran. Dado que el contenido de la expresión infantil no se encuentra alterado, podemos suponer que se trata de una generalización de la estructura gramatical de enunciados que utilizan habitualmente a otros, y por lo tanto que desconocen lingüísticamente la función pronominal.

Otro tipo de error que aparece en los enunciados de Carlos consiste en la adición de los pronombres en locuciones que tanto semántica como estructuralmente están completas [Ej.: (37)].

(37) N: Un niño pequeñito que $m e$ ha tirado a mi la torre mía

Carlos $(2 ; 3)$

El ejemplo anterior muestra como el niño identifica las formas «a mí», «para mí» con los sujetos receptores de la acción (sintácticamente objetos indirectos), mientras que «me» siempre va unido al verbo, identificándolo como una parte del mismo, bien en la forma imperativa («dame»), como en la indicativa («me das»). Por lo tanto funcionalmente establece una diferenciación entre el término «me» y «a mí», que en la lengua española no existe.

Se observa otro tipo de error en el uso de estos términos en la producción lingüística de Carlos. Cuando el niño elabora oraciones más complejas en las que los referentes no son contextuales, bien porque no stán presentes en la situación comunicativa, o porque él mismo inventa para sí otros personajse, aparece sustitución, de forma inadecuada, de unos términos por otros. [Ej.: (38)].

(38) $\mathrm{N}$ : $\mathrm{No} /$ soy una pirata que me he dado un golpe. («me» por «se»).

\section{Carlos $(2 ; 7)$.}

Esto significa, por una parte, carencia en el conocimiento lingüístico, ya que dada su edad y maduración, el niño no es capaz de establecer relaciones fuera de su contexto comunicativo concreto. Por otro lado, aunque ya diferencia los roles conversacionales en los que está inmerso (sobre todo «yo-tú», y los correspondientes «me-te»), todavía no sabe abstraer estos roles, y generalizarlos a otras situaciones no habituales de forma adecuada.

Formas átonas: «la» - «lo» - «le»

Respecto a estos pronombres encontramos inadecuaciones en el uso en determinados enunciados. Así aparecen errores de omisión de términos pronominales en locuciones que exigen su utilización [Ej.: (39), (40)]. 
(39) N: Me ha comprado Toni. (Omisión de «lo»)

Vanesa $(2 ; 6)$

(40) A: Quítamelo

$\mathrm{N}$ : Te quito/ Te quito. (Om. «lo»)

Carlos $(2 ; 2)$

Otro error, observado en las locuciones de estos niños que aparecen también en los pronombres anteriores, es la redundancia, y consiste en la utilización de estos términos en estructuras en las que se expresa de forma explícita el objeto directo o el indirecto y su uso, por tanto se hace innecesario [Ejs.: (41), (42), (43)].

(41) N: No lo veo el bicho.

Vanesa $(2 ; 7)$

(42) N: La ha abierto la nana la puerta.

Celia $(2 ; 4)$

(43) N: Me lo tiraba el cubo con la arena/ me tirada el cu-

bo una niña pequeñita. Carlos $(2 ; 0)$

Esta forma de inadecuación lingüística en los enunciados de estos niños refleja, de alguna manera, que aunque sea habitual el uso de los mismos en sus locuciones verbales, todavía no dominan el significado y las funciones de estos términos, en su totalidad.

Encontramos también, errores en la concordancia entre el pronombe utilizado y el género o el número del referente. En este caso se trata de errores de tipo semántico dado que sólo afecta al contenido, ya que la estructura no sufre ninguna alteración. No obstante, en algunas ocasiones, esta falta de concordancia incide también en los elementos de la oración, afectando de este modo, a la estructura gramatical [Ejs.: (44)-(47)].

(44) N: Lo he tirado yo. (la casa).

(45) N: Si me disparan yo le disparo con una pistola de

Carlos $(2 ; 1)$ verdad.

(46) N: Esto lo tengo sanita. (la boca)

(47) N: Se los ha comido todas.

Carlos $(2 ; 9)$

Celia $(2 ; 7)$

Vanesa $(3 ; 0)$

Por último aparecen formas gramaticalmente incorrectas en la utilización de los deícticos «la» «lo» «le», esto es, en algunas locuciones se sustituye «la» por «le» (laismo), o bien «le» por «lo» (leismo). Dado que estas son formas de uso habituales en los adultos de la comunidad lingüística a la que pertenecen estos niños, no pueden ser considerados errores en el desarrollo del lenguaje infantil.

\section{CONCLUSIONES}

En líneas generales, se observa que la adquisición de los pronombres por parte de los niños, pasa por dos etapas de desarrollo. En un primer momento, estos términos, son utilizados como deícticos, es decir, como recursos comunicativos. Progresivamente su utilización se hace más lingüística. Este hecho se observa claramente a través del uso que los niños hacen de los términos «yotú», ya que sólo en un momento posterior del desarrollo del lenguaje forma parte de enunciados más complejos, mientras que, en un priemr momento, aparecen como elementos únicos de los enunciados, cumpliendo una función de indicación o de señalamiento.

Al mismo tiempo, existe una secuenciación en la adquisición y uso de estos términos en función de la complejidad semántica y sintáctica de los mismos. 
Las primeras formas pronominales que aparecen en las locuciones de estos niños son: «yo» y «tú», cuya utilización es adecuada desde el primer momento coincidiendo con los resultados obtenidos por Clark y Sengl (1978), y Charney (1980). Esto puede deberse, por un lado, a la propia complejidad de los términos en relación con el desarrollo madurativo y cognitivo del niño. Por otro lado, y sobre todo, debido a que son fundamentales en la diferenciación de los roles comunicativos, imprescindibles para el diálogo, cuya adquisición y desarrollo tiene lugar en el período preverbal, ya que el adulto incluye estos términos en la comunicación diádica con el niño. En relación con el hecho de que no aparezcan errores de inversión en el uso de estos pronombres (unido a que tampoco aparecen en los términos «me» y «te») podemos afirmar que estos niños manifiestan un conocimiento evidente de los roles conversacionales (hablante-oyente). La escasa frecuencia de uso de los pronombres nominativos de tercera persona y los plurales, se debe, quizás, a las características propias de la interacción diádica temprana: interpersonal y referida al contexto presente.

Posteriormente, hacia los dos años y dos meses, comienzan a utilizar las formas de dativo y acusativo. Estas formas pronominales tienen funciones eminentemente linguísticas dado que están incluidas en enunciados que necesariamente contienen otros elementos gramaticales (fundamentalmente verbos) por lo cual su utilización está ligada al desarrollo del lenguaje, y probablemente, su aprendizaje tiene lugar como parte del propio verbo. Esto se evidencia por el hecho de que cuando los niños estudiados elaboran mensajes con verbos reflexivos usan adecuadamente los términos «me», y «se», dado que tales verbos implican su presencia en el enunciado; mientras que los errores en la producción aparecen en casos en que los verbos no son reflexivos. Esto ocurre bien por una generalización en el uso de estos pronombres, bien por omisión de los mismos cuando es necesaria su utilizaicón. Además, la niña de medio institucional - con un desarrollo lingǘstico menor - presenta una baja frecuencia de uso de estas formas, y cuando aparecen suelen acompanar, en su mayoría, a verbos reflexivos.

Respecto al uso de los términos «le» «la» «lo», tambén ligados a las formas verbales utilizadas por los niños - en su mayoría transitivas - son las que mayor número de errores presentan y su naturaleza es eminentemente lignüística (omisión, adición, concordancia). Los tipos de errores varían según los sujetos, en el mismo sentido que en el caso anterior, Vanesa presenta errores más graves que Celia y Carlos, esto es, errores de omisión que suponen un desconocimiento mayor de las reglas gramaticales que los de adición y concordancia que aparecen en los otros niños.

Por último, a la vista de los resultados podemos afirmar que la adquisición y evolución de las formas pronominales tiene lugar en los contextos comunicativos. En primer lugar hay que señalar que la adquisición de los mismos es más temprana, y el uso más adecuado que lo que señalan los estudios experimentales dado que los contextos habituales y la interacción con adultos conocidos favorecen la actuación lingǘstica de los niños.

Además se observan diferencias evidentes en el momento de aparición y uso de estos pronombres entre los niños estudiados, en función de su nivel de desarrollo lingǘstico. La niña de medio institucional presenta un uso menos adecuado y más estereotipado, así como una adquisición más tardía, frente a la de los niños de medio familiar, que manifiestan un desarrollo del lenguaje más favorable. 
Otra cuestión que refleja la importancia del contexto en la evolución de los pronombres, es el hecho de que los tipos de errores que aparecen en los enunciados de los niños con niveles de desarrollo lingüístico similar (Carlos y Celia), varía más en función de los sujetos que en función de las edades de los mismos. Esto es, hemos observado que Carlos, aunque aumente la complejidad estructural de sus enunciados a medida que avanza en edad, los errores que produce corresponden a los mismos tipos, al igual que en el caso de Celia. Mientras que si comparamos las locuciones de ambos niños en lo que respecta a la tipología de errores encontramos diferencias. Así aparecen de concordancia de género y número, y de adición en Carlos, mientras que Celia comete fundamentalmente errores de adición y en menor medida de concordancia. Este hecho está más ligado al proceso de enseñanza-aprendizaje infantil que al propio proceso de adquisición del lenguaje.

En el mismo sentido se explicaría la aparición de errores de sustitución de pronombres de tercera persona, definidos como «leismo» y «laismo», que no pueden ser considerados como inadecuaciones en la adquisición de estos términos sino como fruto del contexto de enseñanza-aprendizaje. Esto consituye un uso frecuente en determinadas zonas del Estado Español, y en concreto de los adultos que participan en la interacción con los niños de este estudio.

Así pues, podemos decir, que la adquisición del pronombre es compleja, ya que incluye diversos aspectos del desarrollo infantil como son: competencia comunicativa, cognitiva y del lenguaje. Esto es, el conocimiento de los roles conversacionales, ciertas nociones de la perspectiva del otro y las relaciones establecidas entre las personas y el mundo físico y social, son condiciones necesarias para el proceso interactivo. Al mismo tiempo, la utilización de estos pronombres exige cierto nivel de desarrollo lingǘstico, que se da precisamente gracias al uso del lenguaje. Consecuentemente, la adquisición y desarrollo de las formas pronominales, está enmarcada en el mismo proceso de adquisición del lenguaje $\mathrm{y}$, por lo tanto, tiene lugar en, y gracias a los adecuados contextos interactivos diádicos. En estos contextos interactivos el adulto desempeña un papel fundamental, suple las limitaciones lingüísticas y cognitivas infantiles, haciendo posible y eficaz la comunicación. De esta manera proporciona al niño el contexto en el que contrastar las hipótesis lingüísticas que éste elabora, haciendo posible su adecuado desarrollo.

\section{Referencias}

Alvarez Martinez, M. A. (1989), El Pronombre I: Personales, Artículos, Demostrativos, Posesivos, Madrid, Arco/Libros.

Baltes y Nesselroade, (1981). Métodos de investigación en Psicología Evolutiva: Enfoque del ciclo vital, Madrid, Morata.

Benveniste, (1970), Problemas de Lingüistica general, Madrid, F.C.E.

BüHLer, K. (1968), Teoría del lenguaje, Madrid, F.C.E.

Clark, E. V. y Sengul, C. J. (1978a), «Strategies in the acquisition of deixis» J. Child Language, 5. 457-475.

CLARK, E. V., (1978b), «From gesture to word: on the natural history of deixis in language acquistion", en BRUNER, J. y GARTON, A., Human growth and development, Oxford, Claredom Press.

Charney, R. (1980), «Speech roles and the development of personal pronouns», J. Child Language, $n .^{\circ} 7,509-528$. 
Chist, S. (1981a), «Context-specifity and generalization in the acquisition of pronominal distinctions», J. Child Language, 8. 75-91.

Chiat, S. (1981b), «If were you and you were me: the analysis of pronouns in a pronounreversing child», J. Child Languaje, 9,pp. 359-397.

De Villiers, P. A. y DE Villiers, J. G. (1974). «On this, that, and the other: non-egocentrism in very young children», J. of Experimental Child Psycbology, 18, 438-447.

JAKOBSON, R. (1975), Ensayos de Lingüistica general, Barcelona, Seix Barral.

Muñoz, S. (1986), «Los pronombres», en SIGUAN, M. (Coord), Estudios de Psicolingüística, Madrid, Pirámide.

Lyons, J. C. (1977), Semantics, Cambridge, University Press.

Miller, G. (1974), Lenguaje y Comunicación, Buenos Aires, Amorrortu.

Miller, J. F. (1978), «Evolución de la conducta lingüística de los niños: Enfoque evolutivo del proceso de evaluación», en SCHIEFELBUSH, Bases para la intervención del Lenguaje, Madrid, Alhambra Universidad.

Napolitano, G. (1986), «Genética de la enunciación», en SIGUAN, M. (coord), Estudios de Psicolingǘstica, Madrid, Pirámide.

Netson, K. (1973), «Estructure and estrategy in learning to talk» Monog. Soc. Res. Child Development 38 , Serial 149 .

Peirce, C. (1932). Collected Papers, HOSTHONE, C. y WEISS, P. (ed.) Harward University Press, Cambridge, Mass.

Porto DA Pena, A. (1986), Los Pronombres, Madrid, Edi-G. S. A.

Sнum, G. (1986), Psicolingǘstica aplicada a la adquisición del lenguaje en niños institucionalizados y no institucionalizados, Tesis Doctoral, Madrid, Universidad Complutense.

SHum, G. (1989). «La influencia del contexto de la interacción en la adquisición del lenguaje», Infancia y Aprendizaje, 43, 37-53. 